\title{
Deployable Entry Vehicles for Future Science and Exploration Missions
}

\section{Primary Author:}

Alan Cassell ${ }^{1}$

Entry Systems and Technology Division

NASA Ames Research Center

Phone: 650-604-2325

Email: Alan.M.Cassell@ nasa.gov

\section{Co-Authors:}

Neil Cheatwood ${ }^{2}$

Steven Hughes ${ }^{2}$

Cole Kazemba ${ }^{1}$

Greg Swanson ${ }^{1}$

\section{Endorsers:}

Gerald Lebeau ${ }^{3}$

Michelle Munk ${ }^{2}$

Ethiraj Venkatapathy ${ }^{1}$

Paul Wercinski ${ }^{1}$

Michael Wright ${ }^{1}$

${ }^{1}$ NASA Ames Research Center

${ }^{2}$ NASA Langley Research Center

${ }^{3}$ NASA Johnson Space Center 


\section{EXECUTIVE SUMMARY}

Deployable Entry Vehicle (DEV) technology has made significant advances over the last decade with ground test development campaigns and flight test demonstrations. DEVs offer benefits over traditional rigid entry vehicles including lower volume and mass, while enabling larger payload delivery and greater landing access. A crucial mission benefit is the DEV capability to transform from a compactly stowed configuration within the launch vehicle to a high drag area entry system for delivery of landers, rovers, aerial platforms, and orbiters (via aerocapture). These benefits span the range of mission classes from Small Satellite (smallsat) to human-class exploration systems. This paper will describe DEV technology development status, highlight mission concepts, and recommend future investments.

\section{INTRODUCTION}

NASA's Strategic Space Technology Investment Plan ${ }^{1}$ identified entry, descent and landing (EDL) as one of eight core technology investment areas, and within the EDL core area, DEVs were identified for development. NASA's Space Technology Mission Directorate (STMD) has invested significant resources into maturing two types of DEVs: i) the Hypersonic Inflatable Aerodynamic Decelerator (HIAD) and a mechanically deployable system, ii) Adaptive, Deployable, Entry and Placement Technology (ADEPT). As part of the development of these DEV technologies, new thermal protection system (TPS) materials have been developed and manufactured to enable a flexible TPS for folded stowage in the launch configuration which can then later be deployed into a high drag area aeroshell.

DEVs provide a means by which the ballistic coefficient at entry is relatively unconstrained by the launch configuration (Figure 1). The technologies allow mission implementers to utilize an aeroshell design that fits within existing launch vehicle (LV) shroud (or secondary payload accommodation) volume. Prior to the EDL mission segment, the DEV deploys to form a large diameter, low ballistic coefficient entry configuration. The low ballistic coefficients enabled by DEVs result in significant reductions to the aeroheating and surface pressure experienced during entry which thereby opens up new mission design scenarios not available to missions utilizing traditional rigid capsules. This performance feature has several enabling benefits, including large payload delivery ${ }^{2}$ to the Martian surface or orbit, access to higher altitudes on Mars, and aerocapture missions to Venus and the Ice Giants. ${ }^{3}$ DEVs also provide mission enhancing benefits to a variety of missions including Earth return and smallsat science. $^{4}$

Another key benefit deployable technologies provide is operational flexibility over rigid aeroshells. Since there is no backshell, both HIAD and ADEPT can stow such that access to the payload remains largely unencumbered after integration into LV stack. Facile access to the payload throughout launch vehicle integration simplifies testing and assembly operations. Traditional rigid aeroshell integration sequencing is more difficult, particularly with late access

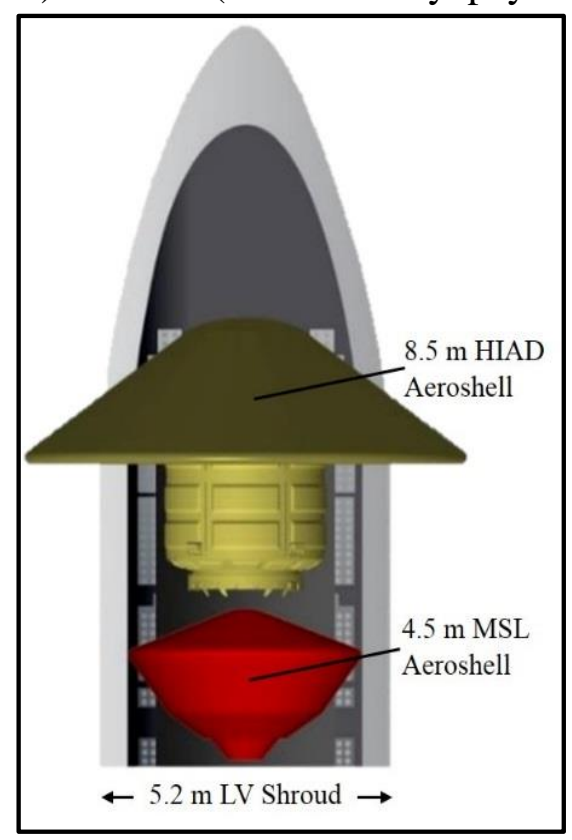

Figure 1 - Illustration of Mars Science Laboratory (MSL) rigid aeroshell maximum diameter constrained by the launch shroud as compared to a deployed HIAD. 
during launch preparations. For example, the installation of radioisotope thermoelectric generators (RTGs) for the Mars Science Laboratory (MSL) and Mars2020 missions required extensive design and testing to integrate an access door in the backshell in order to install the RTG just prior to launch.

International space agencies are also developing DEVs for various atmospheric entry applications. Near-term applications for Earth vicinity and Mars robotic missions are anticipated. The European Space Agency recently funded the European Flexible Heatshield project, EFESTO. ${ }^{5}$ The project will provide advances in thermal control, materials and structures through the design and testing of innovative inflatable TPS solutions for entry vehicles. The Italian Space Agency is also pursuing IRENE (Italian Re-Entry Nacelle), a mechanically deployed entry vehicle slated for a test flight in early $2021 .^{6}$ The IRENE program objectives include demonstrating re-usability for low Earth orbit applications with plans to extend the technology for robotic missions to Mars. The Japanese Aerospace Exploration Agency has been testing deployable entry vehicles for future planetary probe mission applications. ${ }^{7}$ Sub-orbital and orbital test flights have been conducted to understand entry performance. The Chinese Space Agency recently attempted an orbital entry experiment termed as the Flexible Inflatable Cargo Re-entry Vehicle. ${ }^{8}$ The cargo return vehicle is designed to ferry equipment and experiment specimens back to Earth from China's planned space station, which program officials say is set to be completed by 2022. Continued worldwide DEV development activities will undoubtedly see them applied to many different science and exploration missions in the future.

\section{DEPLOYABLE ENTRY VEHICLE OVERVIEW Hypersonic Inflatable Aerodynamic Decelerator (HIAD)}

Atmospheric drag provides the most mass-effective way to decelerate a payload to its target location or capture it into orbit. HIAD technology has been in development for more than 17 years, including many ground test campaigns, two successful suborbital flight tests of a $3 \mathrm{~m} \mathrm{HIAD,} \mathrm{and}$ the upcoming Low-Earth Orbit Flight Test of an Inflatable Decelerator (LOFTID) technology demonstration mission in 2022. The large mass $(1700 \mathrm{~kg})$ and scale (6 $\mathrm{m}$ deployed) of the LOFTID vehicle, combined with the entry environments associated with return from LEO, are relevant to many potential applications at multiple destinations, paving the way for its use on future missions.

HIAD technology is also the leading candidate for the hypersonic phase of EDL for NASA's human Mars exploration studies. To date, all missions to the Martian surface have employed rigid capsules which have a maximum delivered

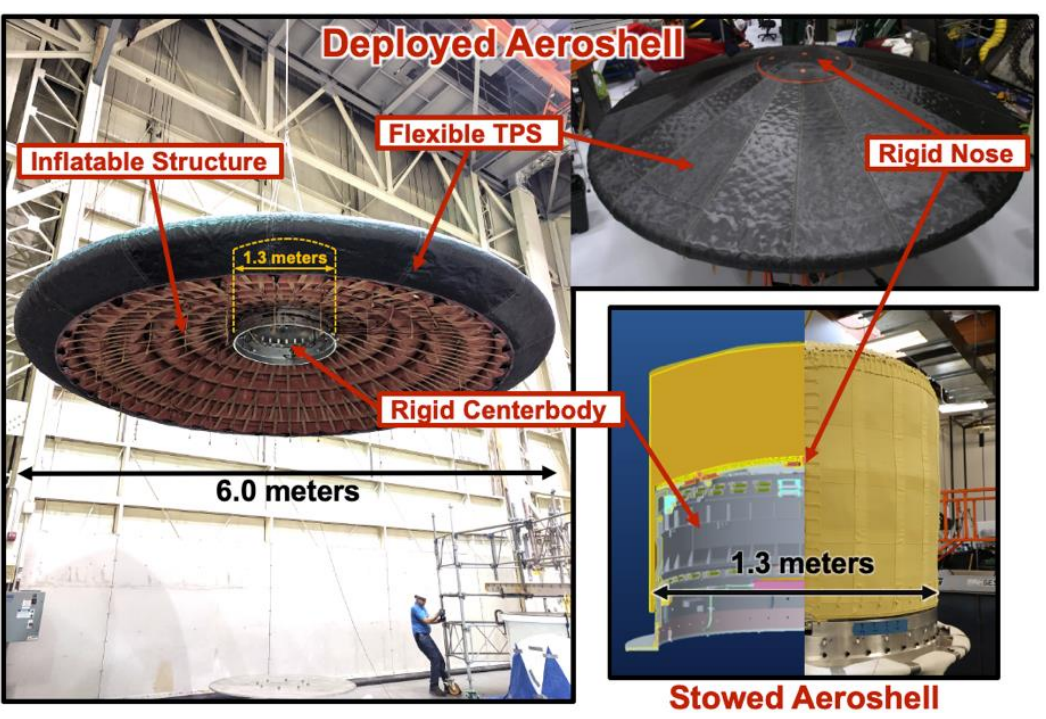

Figure 2 - Deployed forward and aft views HIAD showing the flexible TPS and inflatable structure. The aeroshell compactly stows in front of the payload area to allow facile access. payload capability of 1.5 tons. 
For Human Mars EDL, the estimated requirement is 20 tons of usable payload to the Martian surface, requiring an 18 m HIAD. ${ }^{9}$ To progress toward this need, the next step is to demonstrate HIAD technology at a $12 \mathrm{~m}$ scale. Current technology development has already produced components at this scale.

The HIAD aeroshell consists of two main components: the inflatable structure that maintains the aeroshell shape against the aerodynamic drag forces, and a protective flexible TPS (FTPS) that withstands the thermal loading. The term "flexible" refers to the FTPS being foldable, packable, deployable, and tailorable - the FTPS contains woven ceramic fibers on the outer layer and is not elastic. The FTPS protects the forward surface of the inflatable structure, and if needed, can also be employed on the aft surface of the inflatable structure and the payload. The inflatable structure is a stacked toroid assembly anchored to a rigid centerbody and nose that can be scaled to meet a mission's payload requirements.

NASA has developed a suite of material sets that can be tailored to the mission's expected thermal environments. HIAD uses cutting edge high-strength, low-weight materials allowing the deployable aeroshell (FTPS and inflatable structure) to react roughly $75 \mathrm{x}$ its weight in aerodynamic loading. For reference, the LOFTID $6 \mathrm{~m}$ diameter deployable aeroshell weighs approximately $400 \mathrm{lbs}(180 \mathrm{~kg})$ and the combined system is capable of reacting $\sim 30,000 \mathrm{lbs}$ (133.4 $\mathrm{kN}$ ) of pressure load. In flight the aeroshell is deployed prior to atmospheric entry using the inflation system which fills the tori to their operating pressure. To date, blowdown systems of compressed nitrogen have been employed. Gas generation systems are under development which will reduce overall system mass.

Taking advantage of the adaptable nature of soft good materials and fabrication techniques, inflatable structure geometries and features can be incorporated into HIAD designs to provide flight control authority. Generating these asymmetric shapes and features enables HIAD technology flight performance to be expanded to meet a much broader range of planetary missions that require precision landing or control, such as Mars, Titan and Earth return missions. Trajectory analysis and flight simulations show that a lift to drag ratio (L/D) of 0.15 provides sufficient control authority to meet required targeting accuracy and reduce dependency upon propulsion systems during Mars entry and descent. ${ }^{10}$ This approach can also be implemented such that the aerodynamic features provide independent down range and cross range control with rapid response times for actuation. The preferred approach for HIAD guidance and control is to use shape morphing to deform the HIAD from its symmetrical shape to create a nonuniformity for force control. This can be accomplished by morphing the stacked torus
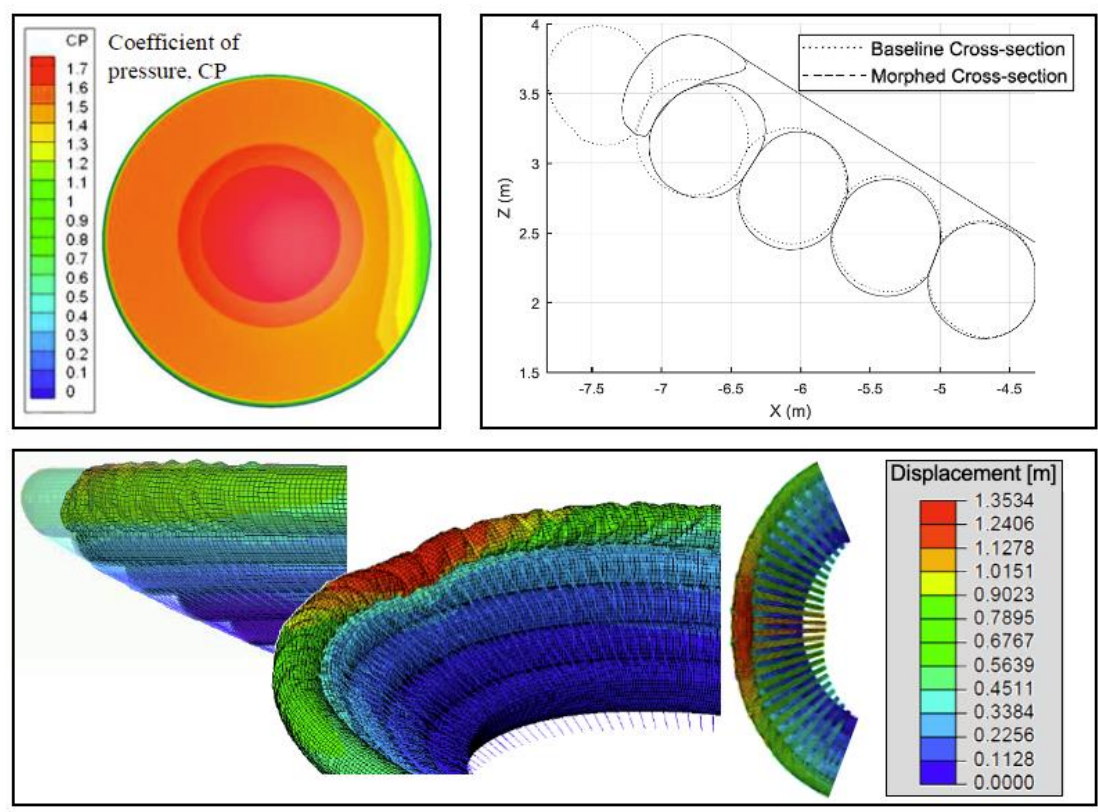

Figure 3 - HIAD shape morphing approach to achieve guided entry for precision targeting and landing. 
structure with actuators to modulate the lift and sideslip angles during entry and descent. This can be accomplished by independently displacing quadrants of the structure as shown in Figure 3 . With active shape control, actuators provide quick response to modulate the amount of shape change required. Separate lift and sideslip commands achieve independent actuation for each quadrant of the aeroshell to guide the vehicle towards the target point.

Following the completion of the LOFTID experiment, HIAD technology will have been demonstrated in Earth orbital entry environments at a scale relevant to many New Frontiers and Discovery class science missions. The next step will be to demonstrate guided flight. Additional capability is achievable with further investment in the areas noted in the recommendations section. The maturity of the technology, the mission benefits it provides, and its position as a key technology investment for enabling Human Mars EDL makes it an attractive candidate for mission infusion in the coming decade.

\section{Adaptive Deployable Entry \& Placement Technology (ADEPT)}

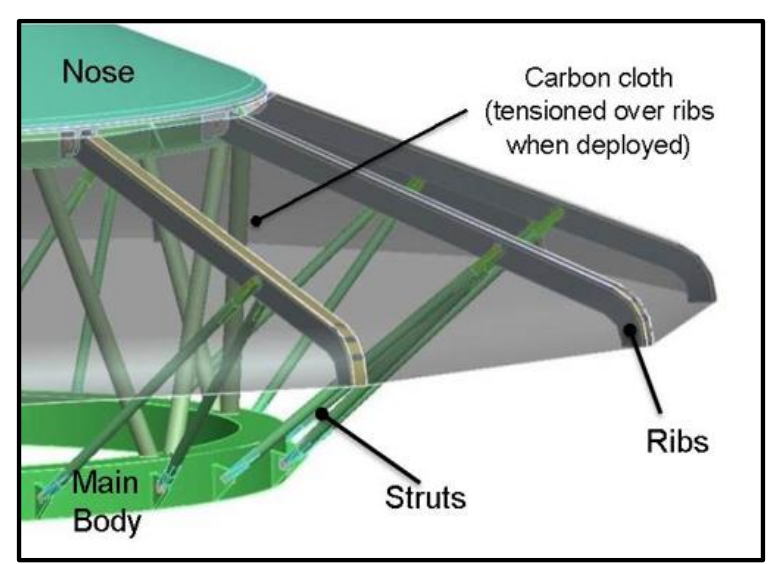

Figure 4 - The key components that comprise the ADEPT DEV system.

ADEPT is a mechanically deployed entry system that provides an alternative to traditional high ballistic coefficient rigid aeroshells. A stowed release mechanism removes the constraints on the ribs allowing the structure to begin deployment, similar to how an umbrella deploys. In general, the ADEPT structural skeleton is made up of four primary structural elements: main body, nose cap, ribs, and struts. An example of this particular arrangement is shown in Figure 4. The main body consists of lower and upper rings that are connected by a truss structure. The main body lower ring is a box section that supports the lower ends of the rib support struts and serves as the attachment interface to a spacecraft or secondary payload adapter. The main body upper ring (supported by the main body struts) acts as the attach/latch location for the nose cap ring. In alternate embodiments, the main body structure (and/or deployment mechanism) can be incorporated as part of the payload interface. The ADEPT structural skeleton is constrained at the ribs during launch to prevent excessive motion in response to the vibroacoustic environment. Figure 5 shows stowed and deployed configurations of a $2 \mathrm{~m}$ diameter test article.

The key technology enabler for ADEPT is the flexible multilayer 3-d woven carbon fabric that forms a semi-rigid membrane when pre-tensioned by deployment of supporting ribs. This multilayered woven fabric transfers aerodynamic loads to the support structure while operating at very high temperatures induced by

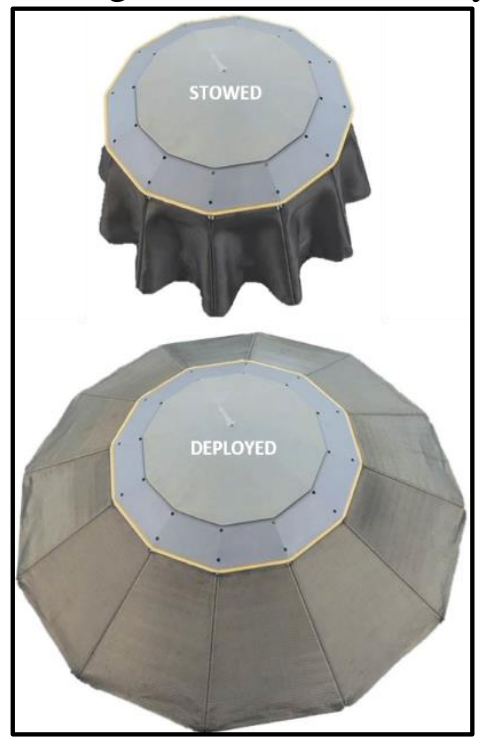

Figure 5 -2 m diameter ADEPT deployment test article in the stowed \& deployed configurations. 


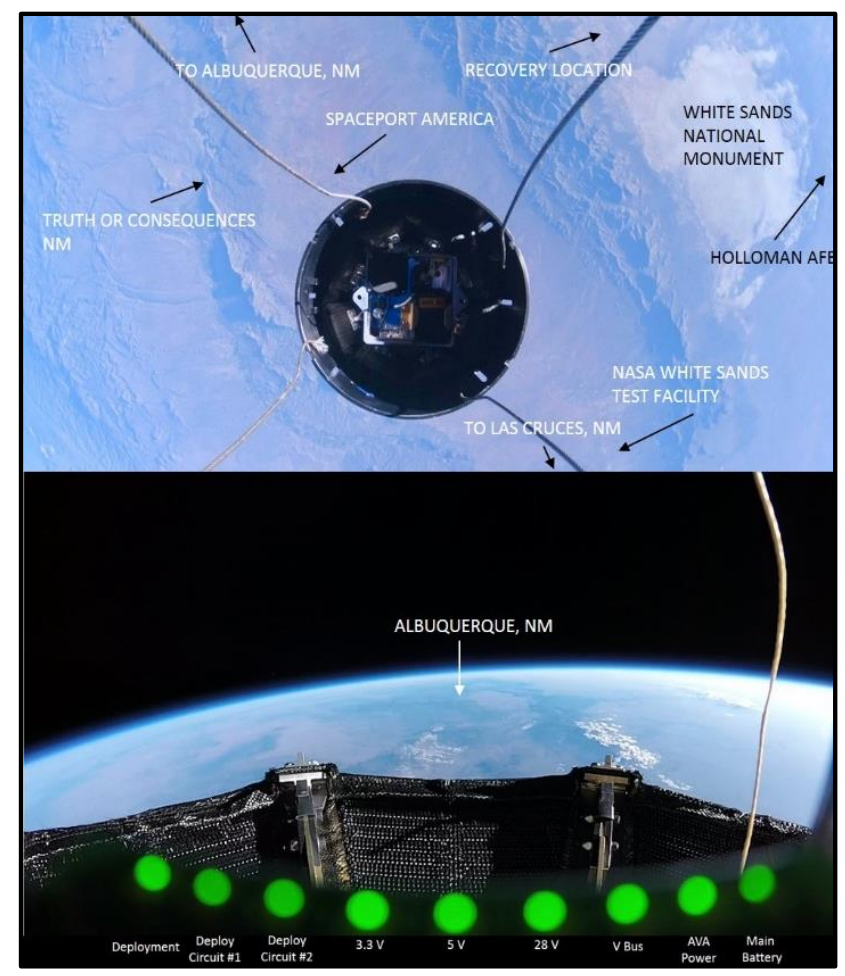

Figure 6 - The top panel shows the stowed ADEPT inside the payload module of the $\mathrm{LV}$ and the bottom panel an on-board camera view of the deployed configuration just prior to reaching apogee.

entry aeroheating. The bottom layers of the fabric carry the aerodynamic load while the top layers manage the thermal energy. Ground testing campaigns have demonstrated the ability of the carbon fabric to withstand combined mechanical and aerothermal loads. System level aerothermal tests have demonstrated entry environments relevant for multiple destinations including Venus, Mars, Titan, Uranus, Neptune, and Earth return. ${ }^{11}$

Recently, a small spacecraft class $(0.7 \mathrm{~m}$ deployed diameter) version of ADEPT was tested in a sub-orbital flight experiment (Figure 6). ${ }^{12}$ The ADEPT Sounding RocketOne (SR-1) flight experiment is a component of a broader $1 \mathrm{~m}$-class development strategy for mission infusion of smallsat-class payloads. The SR-1 flight test matured ADEPT in the areas of deployment and structural integrity for small-class missions. It provided key aerodynamic performance data of the unique ADEPT geometry with its faceted heatshield and open-back configuration. In addition, SR-1 was an integrated system demonstration in a partially relevant environment (SR-1 did not experience significant heating but reached supersonic speeds). Given the current maturation and development of the ballistic (non-lifting) $1 \mathrm{~m}$-class ADEPT, the next step in ADEPT maturation is to focus on configurations that enable aerocapture and precision landing mission capabilities. ADEPT is particularly attractive for evaluating various guidance and control approaches as the deployable structure enables attachment points for various actuation methods such as control surfaces, moving mass elements, or RCS thrusters. The Pterodactyl Project is analyzing various ADEPT configurations to study the efficacy of guidance and control architectures for DEVs. ${ }^{13}$

\section{MISSION APPLICATIONS}

DEVs can enhance, and even enable, science missions by providing the capability to deliver a payload to higher elevation landing sites (e.g. - Southern Highlands at Mars), and/or greater payload mass and volume than has been demonstrated by rigid capsule systems to date. ${ }^{14}$ DEVs are well suited to address the stated emphasis on technology development for aerocapture, drag modulation, and advancements in TPS materials. ${ }^{15}$

HIADs have mission applicability at Earth, providing for International Space Station downmass, enabling return for free-flying orbital manufacturing, and the recovery of spent launch vehicle assets for reuse. United Launch Alliance is partnering with NASA to recover their first stage Vulcan booster, which can reduce the overall cost of access to space. ${ }^{16}$ Use of HIADs can also support Goal IV in the Mars Exploration Planetary Analysis Group 2020 Goals Document which proposes efforts to prepare for human exploration. ${ }^{17}$ Employing a HIAD for a science 
mission would provide a great synergy with the Human Mars EDL development and offer a compelling advantage against competing mission proposals.

A primary mission infusion opportunity for ADEPT in the coming decade is for use in performing drag modulated aerocapture. Drag modulated aerocapture uses in-flight transformations of an entry vehicle's drag area to control the amount of deceleration produced during an atmospheric pass. This could revolutionize the capabilities of small spacecraft in planetary science, and also feed forward to future outer planet missions. Aerocapture is particularly well-suited for small spacecraft orbit insertion, due to the difficulty of designing and integrating a propulsion system to perform hundreds to thousands of meters per second delta-V within the constraints of smallsat design parameters. Further details on drag modulated aerocapture can be found in Austin et al..$^{18}$

\section{CONCLUSIONS}

- DEVs offer an alternative to rigid aeroshell approaches with the primary benefit of not being constrained by the launch vehicle payload volume.

- Space agencies across the globe are investing in the development of DEVs for future Human and robotic exploration.

- The upcoming LOFTID orbital flight technology demonstration mission will demonstrate the largest blunt body aeroshell ever flown, with feed forward to Mars missions.

- HIAD-based DEVs are the leading EDL technology for large landed payload mass (>20 t) landers at Mars required for Human exploration.

- ADEPT utilizes a high performance $3 \mathrm{~d}$ woven carbon fabric thermal protection system that can be utilized at multiple destinations in the Solar system.

- ADEPT offers multiple embodiments, including as a detachable drag skirt for drag modulated aerocapture and the potential to integrate control systems on the rigid portion of the deployed aeroshell.

\section{RECOMMENDATIONS}

- Include HIAD technology in the Decadal Survey as an approach for landing large payloads for robotic- and human-class missions to Mars. Synergy with Mars robotic exploration should be considered within the context of the overall Solar System exploration strategy.

- Investigate DEVs for aerocapture-based orbit insertion missions to Venus, Titan and the Ice Giants. ADEPT is particularly attractive for small spacecraft class aerocapture missions for Venus, with potential feed forward to Ice Giant missions.

- Continue investments in high-temperature structures and flexible TPS to improve DEV aerothermodynamic, structural, and thermal protection performance capability.

- Invest in novel atmospheric entry control system technology development for guided DEVs to support missions requiring precision landing, such as human-class missions to Mars, and/or narrow entry corridors.

- Advocate for technology demonstration missions to be included as secondary payloads on future launch opportunities as a means to advance DEV technology while also providing the opportunity for valuable science return.

- Engage with the DEV technology teams early during the mission concept development phase to understand DEV benefits and define achievable atmospheric entry envelope for that application space and mission benefits. 


\section{REFERENCES}

1. NASA STMD Strategic Technology Investment Plan, https://www.nasa.gov/sites/default/files/atoms/files/2017-8-1_stip_final-508ed.pdf

2. Dwyer-Cianciolo, A. et al "Human Mars Entry, Descent and Landing Architecture Study: Deployable Decelerators" AIAA SPACE Forum, 2018-5191.

3. Austin, A. et al "SmallSat Aerocapture: Breaking the Rocket Equation to Enable a New Class of Planetary Missions" $70^{\text {th }}$ International Aeronautical Congress, IAC19.B4.8.2x51457, 2019, Washington, DC.

4. Cassell, A. M. et al "ADEPT, A Mechanically Deployable Re-Entry Vehicle System, Enabling Interplanetary CubeSat and Small Satellite Missions" $32^{\text {nd }}$ Annual AIAA/USU Conference on Small Satellites, 2018, SSC18-XII-08.

5. Bonetti, D. et al "European Flexible Heat Shields: Advanced TPS Design and Tests for Future In-Orbit Demonstration (EFESTO)" FAR 2019, Monopoli, Italy.

6. Vernillo, P. et al "Mini-IRENE Flight Experiment - Stepwise Qualification Approach of a Deployable and Flexible Heat Shield for An Orbital Re-Entry Mission” FAR 2019, Monopoli, Italy.

7. Yamada, K. et al "Nano-satellite "EGG" for Deployment Demonstration of Membrane Aeroshell for Future Planetary Probe", $14^{\text {th }}$ International Planetary Probe Workshop, 2017.

8. https://spaceflightnow.com/2020/05/06/experimental-chinese-cargo-return-capsulemalfunctions-during-re-entry/

9. Dwyer-Cianciolo, A. M. et al "Human Mars Entry, Descent and Landing Architecture Study: Phase 3 Summary" AIAA SciTech Forum, Orlando, FL, 2020-1509.

10. Dwyer-Cianciolo, A. M, Powell, R. W. "Entry Descent and Landing Guidance and Control Approaches to Satisfy Mars Human Mission Landing Criteria" AAS/AIAA Space Flight Mechanics Conference, 2017.

11. Cassell, A. M. et al "System Level Aerothermal Testing for the Adaptive Deployable Entry and Placement Technology (ADEPT)" $13^{\text {th }}$ International Planetary Probe Workshop, 2016.

12. Cassell, A. M. et al "ADEPT Sounding Rocket One Flight Test Overview" AIAA Aviation 2019 Forum, 2019-2896, Dallas, TX, 2019.

13. D'Souza, S. N. et al "Developing an Entry Guidance and Control Design Capability Using Flaps for Lifting Nano-ADEPT” AIAA Aviation 2019 Forum, 2019-2901.

14. Cheatwood, F. M. et al "Manufacturing Challenges and Benefits when Scaling the HIAD Stacked-Torus Aeroshell to a 15 m-Class System" IEEE Aerospace Conference, Big Sky, MT, 2016, pp. 1-13, doi:10.1109/AERO 2016.7500773.

15. Spilker, L. J. et al "Exploration Strategy for the Outer Planets 2023-2032: Goals and Priorities (Updated). Outer Planets Assessment Group White Paper, 2020.

16. Reed, J. G. et al "Performance Efficient Launch Vehicle Recovery and Reuse" AIAA SPACE Forum, 2016-5321.

17. MEPAG (2020) "Mars Scientific Goals, Objectives, Investigations, and Priorities: 2020. D. Banfield, ed., 89 p. white paper posted March, 2020 by the Mars Exploration Program Analysis Group (MEPAG) at https://mepag.jpl.nasa.gov/reports.cfm

18. Austin, A. et al "Enabling and Enhancing Science Exploration Across the Solar System: Aerocapture Technology for SmallSat to Flagship Missions" White Paper for the Planetary Decadal Survey, 2023-2032. 